\title{
Impact of Acetazolamide, a Carbonic Anhydrase Inhibitor, on the Development of Intestinal Polyps in Min Mice
}

\author{
Nobuharu Noma ${ }^{1,2}$, Gen Fujii ${ }^{3}$, Shingo Miyamoto ${ }^{4}$, Masami Komiya ${ }^{4}$, Ruri Nakanishi ${ }^{4}$, \\ Misato Shimura ${ }^{1,2}$, Sei-ichi Tanuma ${ }^{2}$ and Michihiro Mutoh 1,3,4,* \\ 1 Division of Cancer Prevention Research, National Cancer Center Research Institute, 5-1-1 Tsukiji, Chuo-ku, \\ Tokyo 104-0045, Japan; nnoma1015@gmail.com (N.N.); misato.shimura@gmail.com (M.S.) \\ 2 Division of Biochemistry, Faculty of Pharmaceutical Sciences, Tokyo University of Sciences, 2641 Yamazaki, \\ Noda-shi, Chiba 278-8510, Japan; tanuma@rs.noda.tus.ac.jp \\ 3 Division of Carcinogenesis and Cancer Prevention, National Cancer Center Research Institute, 5-1-1 Tsukiji, \\ Chuo-ku, Tokyo 104-0045, Japan; gfujii@ncc.go.jp \\ 4 Epidemiology and Prevention Division, Research Center for Cancer Prevention and Screening, \\ National Cancer Center, 5-1-1 Tsukiji, Chuo-ku, Tokyo 104-0045, Japan; shinmiya@ncc.go.jp (S.M.); \\ mkomiya@ncc.go.jp (M.K.); rnakanis@ncc.go.jp (R.N.) \\ * Correspondence: mimutoh@ncc.go.jp; Tel.: +81-03-3542-2511 (ext. 4351)
}

Academic Editors: Takuji Tanaka and Masahito Shimizu

Received: 10 March 2017; Accepted: 12 April 2017; Published: 17 April 2017

\begin{abstract}
Colorectal cancer is a common cancer worldwide. Carbonic anhydrase (CA) catalyzes the reversible conversion of carbon dioxide to bicarbonate ion and a proton, and its inhibitor is reported to reduce cancer cell proliferation and induce apoptosis. Therefore, we asked whether acetazolamide, a CA inhibitor, could inhibit intestinal carcinogenesis. Five-week-old male Apc-mutant mice, Min mice, were fed a AIN-76A diet containing 200 or 400 ppm acetazolamide. As a result, acetazolamide treatment reduced the total number of intestinal polyps by up to $50 \%$ compared to the control group. In addition, the acetazolamide-treated group had low cell proliferation and a high apoptosis ratio in the intestinal polyp epithelial cells. Moreover, the mRNA expression level of proinflammatory cytokines, such as $I L-6$, involved in the cell proliferation was decreased in the polyp part of the acetazolamide-treated group. Next, we examined the effects of acetazolamide on the activation of several transcriptional factors (AP-1, HIF, HSF, NF- $\mathrm{kB}$, NRF2, p53, and STAT3) using a reporter gene assay in human colon cancer cells, Caco-2 cells. Among the examined transcriptional factors, NRF2 transcriptional activation was strongly induced. NRF2-targeting genes, $\gamma G C S, G P x 1$, HO-1, and NQO-1, were also elevated in the intestinal polyps of acetazolamide-treated Min mice. Our results suggested that $\mathrm{CA}$ is involved in intestinal carcinogenesis. Acetazolamide could inhibit polyp formation through suppressing local/general cytokine levels, i.e., IL-6, via NRF2 activation.
\end{abstract}

Keywords: carbonic anhydrase; acetazolamide; NRF2; IL-6; colorectal cancer chemoprevention

\section{Introduction}

Colorectal cancer (CRC) is a common cancer worldwide. Approximately 1.4 million new CRC cases occurred in 2012, and the incidence is assumed to increase to 2.4 million/year by 2035 [1]. Despite the successive development of anti-cancer agents, the mortality from CRC remains high. Therefore, a new strategy for controlling the development of cancer, such as using chemopreventive agents, is in great demand [2].

Carbonic anhydrase (CA) catalyzes the reversible conversion of carbon dioxide to bicarbonate ion and a proton, and CA is found in many types of organs in humans. This zinc-containing metalloenzyme 
plays important roles in many physiological processes, including $\mathrm{pH}$ and $\mathrm{CO}_{2}$ homeostasis and calcification [3]. There are 16 types of CA isozymes, including the cytoplasmic type (CAI, II, III, VII, and XIII); membrane-bound type (CAIV, IX, XII, XIV, and XV); mitochondrial type (CAVa and Vb); and secreted type (CAVI) [3,4]. Some CA isozymes are reported to be associated with carcinogenesis and tumor progression. For instance, two hypoxia-inducible CA isozymes, CAIX and CAXII, promote tumor growth through regulating the $\mathrm{pH}$ in the tumor microenvironment $[5,6]$. CAII is ubiquitously expressed across normal and tumor tissue-only CAIX and CAXII are induced in tumors [7]. It has been reported that $\mathrm{CA}$ inhibitors could significantly reduce cell proliferation and induce apoptosis in human cervical cancer HeLa cells and human renal cell adenocarcinoma 786-O cells along with decreasing the intracellular $\mathrm{pH}$ [8]. More relevant to the current study, it was recently reported that PEGylated bis-sulfonamides, CA inhibitors, can inhibit the growth of colorectal cancer cells, HT-29 [9].

Acetazolamide is a heterocyclic primary sulfonamide that can inhibit CA. Acetazolamide is an old medication used to treat edema, heart failure, mountain sickness, Meniere's disease, and more. For cancer cells, it has been reported that acetazolamide could significantly reduce cell proliferation and induce apoptosis in human cancer cells [8]. In an in vivo setting, the administration of acetazolamide $(40 \mathrm{mg} / \mathrm{kg} / \mathrm{d})$ for 21 days dramatically reduced the number of lung metastases (inhibition rate of lung metastases $=83.9 \%$ ) in a well-characterized Lewis lung carcinoma model [10]. However, the effects of acetazolamide on intestinal carcinogenesis have not yet been clarified.

Therefore, in the present study, we examined the effects of acetazolamide on intestinal polyp development in Apc-mutant Min mice. The Min mouse is an animal model for human familial adenomatous polyposis [11,12]. The mouse has a mutation in codon 850 of the Apc gene and resultant activation of $\beta$-catenin signaling causes $\sim 100$ intestinal polyps (adenoma), mainly in the small intestine. We also examined the effects of acetazolamide on the transcriptional activation of NF-E2-related factor 2 (NRF2) and expression of B-cell lymphoma 2 (Bcl-2). Based on our results, we also discuss the mechanisms involved in the suppressive effects of acetazolamide in Min mice.

\section{Results}

\subsection{Suppression of Intestinal Polyp Formation in Min Mice by Acetazolamide Treatment}

Treatment with 200 ( $0.6 \mathrm{mg} /$ day/mouse) or $400 \mathrm{ppm}$ (1.2 mg/day/mouse) acetazolamide for 8 weeks to Min mice did not affect the body weight, food intake, or clinical signs compared to untreated mice throughout the experimental period. Table 1 summarizes data for the number and distribution of intestinal polyps in the untreated control $(0 \mathrm{ppm})$ and 200 and $400 \mathrm{ppm}$ acetazolamide-treated groups. Treatment with $200 \mathrm{ppm}$ acetazolamide reduced the total number of polyps to $72.1 \%$ of the untreated control value. Of note, the reduction in the middle sections of the intestines was $54.1 \%(p<0.05)$ in the acetazolamide group. Treatment with $400 \mathrm{ppm}$ acetazolamide significantly reduced the number of polyps in all parts of the intestine, and the total number of polyps was reduced to $53.7 \%(p<0.05)$ of the untreated control value.

Table 1. The number of intestinal polyps/mouse in Min mice treated with or without acetazolamide.

\begin{tabular}{ccccccc}
\hline \multirow{2}{*}{ Dose (ppm) } & \multirow{2}{*}{ Number of Mice } & \multicolumn{5}{c}{ Number of Polyps/Mouse } \\
\cline { 3 - 5 } & & \multicolumn{3}{c}{ Small Intestine } & \multirow{2}{*}{ Colon } & \multirow{2}{*}{ Total } \\
\cline { 3 - 5 } & & Proximal & Middle & Distal & & \\
\hline 0 & 10 & $4.5 \pm 1.5$ & $17.0 \pm 9.7$ & $26.8 \pm 9.8$ & $0.5 \pm 1.0$ & $48.8 \pm 18.8$ \\
200 & 10 & $3.9 \pm 1.8$ & $9.2 \pm 3.2^{*}$ & $21.8 \pm 6.3$ & $0.3 \pm 0.7$ & $35.2 \pm 9.2$ \\
400 & 10 & $2.7 \pm 1.2^{*}$ & $8.0 \pm 2.3^{*}$ & $15.4 \pm 6.2^{* *}$ & $0.1 \pm 0.3$ & $26.2 \pm 7.8^{* *}$ \\
\hline
\end{tabular}

Data are presented as the means \pm SD. Significantly different from the untreated control group at $p<0.05$ and ${ }^{* *} p<0.01$.

To evaluate the suppressive effects of acetazolamide on intestinal polyp development in Min mice, proliferating cell nuclear antigen (PCNA) was stained in the cell nuclei by immunohistochemistry. The percentage of PCNA-positive cells in each polyp was significantly reduced by acetazolamide 
treatment from $51.0 \%$ (0 ppm) to $28.7 \%$ (400 ppm) (Figure 1A). To assess the cell growth inhibition mechanisms in response to acetazolamide, several cell growth-related molecules were analyzed by quantitative RT-PCR. Downregulation of the c-Myc and cyclinD1 expression levels in the small intestinal polyps of Min mice was apparent compared with the untreated group (Figure 2A,B).

A

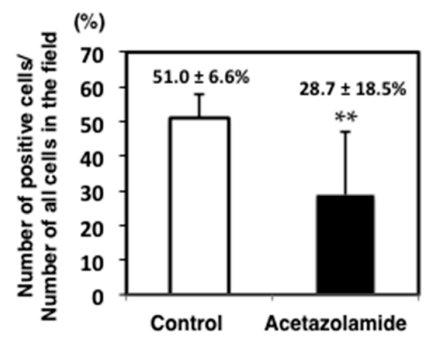

B

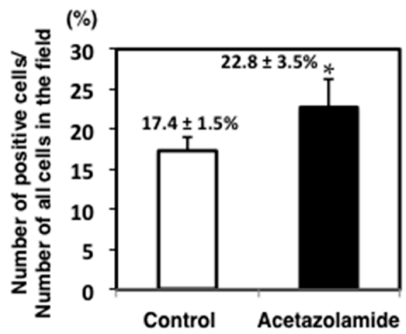

Figure 1. Change in the cell cycle-related and apoptosis-related indexes in intestinal polyps with and without acetazolamide treatment. Immunohistochemistry of proliferating cell nuclear antigen (PCNA) (A) and single-stranded DNA (ssDNA) (B) was performed in a polyp of the small intestine of a Min mouse treated with $400 \mathrm{ppm}$ of acetazolamide and control treatment. The rates of positive cells were calculated by the number of positive cells over all cells in the field. Data are given as the mean \pm SD (No. of polyp $=5$ ). ${ }^{*} p<0.05,{ }^{* *} p<0.01$ vs. untreated control.

A

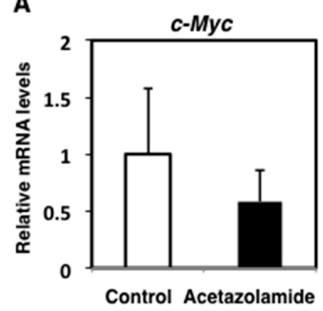

C
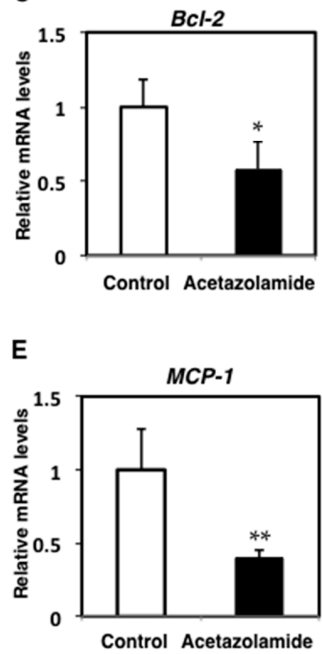

B

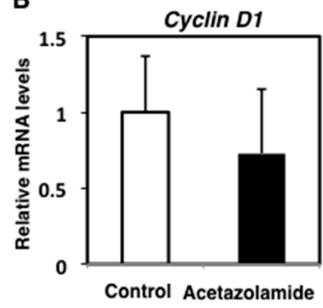

D
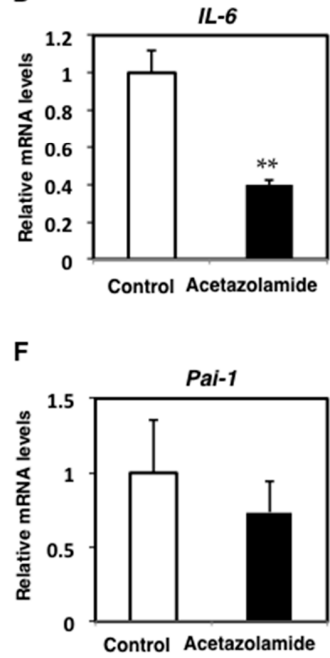

Figure 2. Relative expression levels of cell cycle-related factors, apoptosis-related factors, or proinflammation-related factors in intestinal polyps with and without acetazolamide treatment. Quantitative real-time PCR analyses were performed to determine the c-Myc (A), cyclin D1 (B), B-cell lymphoma 2 (Bcl-2) (C), interleukine-6 (IL-6) (D), monocyte chemoattractant protein-1 (MCP-1) (E), and plasminogen activator inhibitor-1 (Pai-1) (F) mRNA expression levels in the polyps of Min mice with or without $400 \mathrm{ppm}$ of acetazolamide. Values were set at 1.0 in the untreated controls and relative levels are expressed as the mean $\pm \mathrm{SD}(n=5)$. Glyceraldehyde-3-phosphate dehydrogenase (GAPDH) mRNA was used to normalize the data. ${ }^{*} p<0.05,{ }^{* *} p<0.01$ vs. untreated control. 
To investigate the effect of acetazolamide on apoptosis in intestinal epithelial cells, the intestinal polyps of Min mice were immunohistochemically stained with anti-ssDNA (single-stranded DNA) antibody. The rates for ssDNA positive cells in the intestinal polyps were significantly increased by acetazolamide treatment from $14.7 \%(0 \mathrm{ppm})$ to $22.8 \%(400 \mathrm{ppm})(p<0.05)$ (Figure 1B). To assess the cell apoptosis induction mechanisms with acetazolamide, antiapoptotic factor $\mathrm{Bcl}-2$ was analyzed by real-time quantitative reverse transcription polymerase chain reaction (qRT-PCR). The mRNA expression level of $\mathrm{Bcl}-2$ was decreased to $57.7 \%(p<0.05)$ in intestinal polyps compared to the untreated group (Figure 2C).

\subsection{Suppression of Inflammatory Cytokine mRNA Levels in the Intestinal Polyps and Liver in Min Mice Treated with Acetazolamide}

To confirm the expression pattern of acetazolamide targeted molecules in Min mice, the expression levels of the inflammatory cytokines, interleukine-6 (IL-6), monocyte chemoattractant protein-1 (MCP-1), and plasminogen activator inhibitor-1 (Pai-1), were examined by qRT-PCR. The mRNA expression levels of $I L-6$ and $M C P-1$ were significantly decreased $(p<0.01)$ with the $400 \mathrm{ppm}$ acetazolamide treatment compared to the untreated group (Figure 2D,E). The expression level of Pai-1 mRNA was decreased to $73.6 \%$ with acetazolamide treatment (Figure $2 \mathrm{~F}$ ).

To investigate the effect of acetazolamide on other organs, the expression levels of hepatic $I L-6$, MCP-1, and Pai-1 in Min mice were examined by qRT-PCR. The mRNA expression levels of $I L-6$, MCP-1, and Pai-1 in the liver were downregulated by $48.9 \%, 81.4 \%$, and $34.4 \%$, respectively, with the $400 \mathrm{ppm}$ acetazolamide treatment compared to the untreated group (Figure 3). In serum, the IL-6 concentration tended to decrease with acetazolamide treatment (Figure 4).
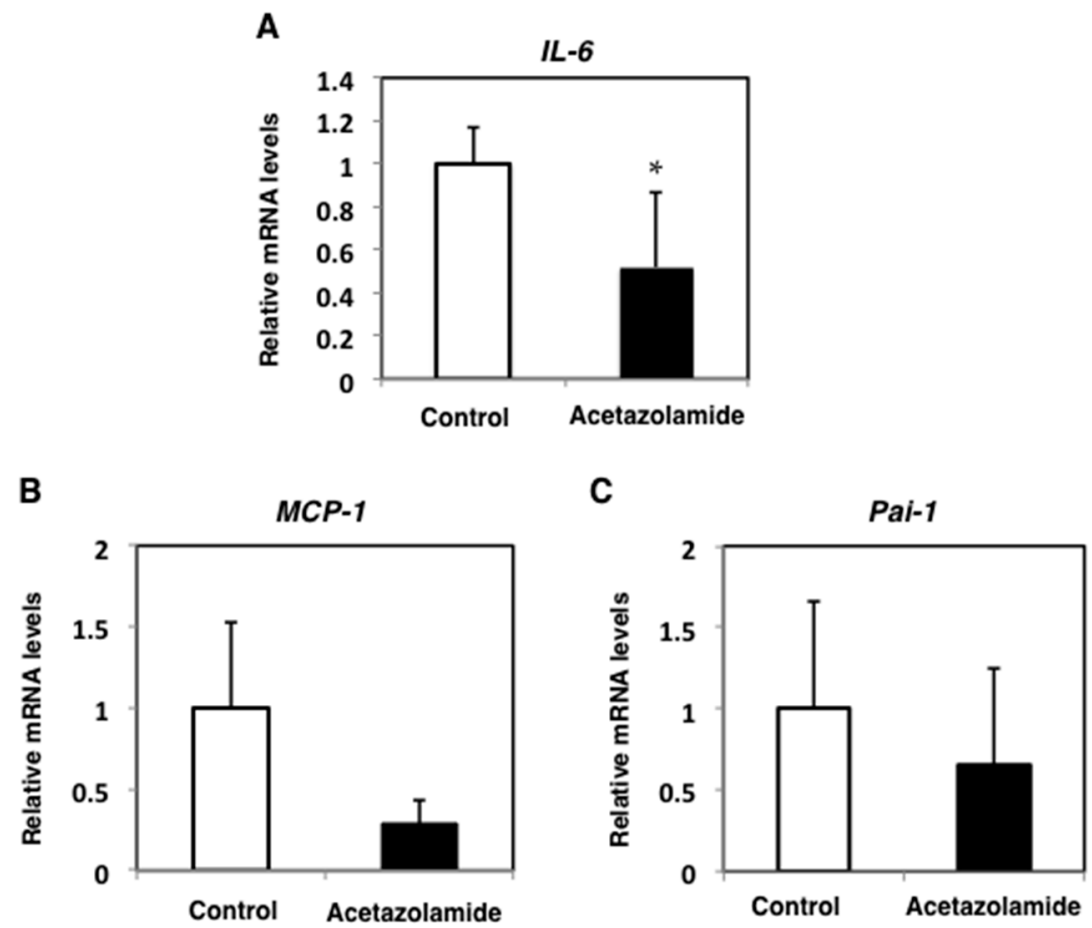

Figure 3. Relative expression levels of inflammatory cytokines IL-6 (A); MCP-1 (B); and Pai-1 (C) in the livers of Min mice with acetazolamide treatment. The data are given as the mean $\pm \operatorname{SD}(n=4)$. * $p<0.05$ vs. untreated control. 


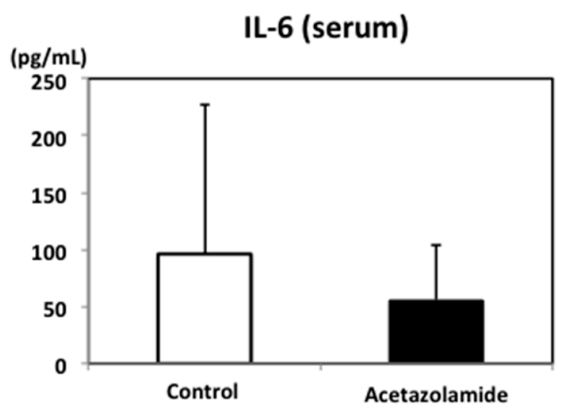

Figure 4. Effects of the serum IL-6 levels in Min mice with acetazolamide treatment. Murine serum IL-6 levels were measured using a Mouse IL-6 Quantikine ELISA Kit (R\&D Systems, Minneapolis, MN, USA) according to the manufacturer's protocol. The data are given as the mean $\pm \operatorname{SD}(n=5)$.

\subsection{Effects of Acetazolamide on Seven Oxidative Stress-Related Transcriptional Activities in Human Colorectal Cancer Cells}

The effects of acetazolamide on oxidative stress-related transcriptional activity were evaluated in Caco-2 cells. The activator protein-1 (AP-1), hypoxia inducible factor (HIF), histamine sensitizing factor (HSF), nuclear factor-kB (NF-kB), NRF2, p53, and signal transducer and activator of transcription 3 (STAT3) transcriptional activities were tested after $24 \mathrm{~h}$ of $500 \mu \mathrm{M}$ acetazolamide treatment. NRF2 activity was significantly increased by acetazolamide (Figure 5A). These activities were also tested on other human colon cancer cell lines, SW48 and HCT15 cells, and acetazolamide treatment increased the NRF2 activity 1.3- (Figure 5B) and 1.2-fold (Figure 5C), respectively, in these cells.

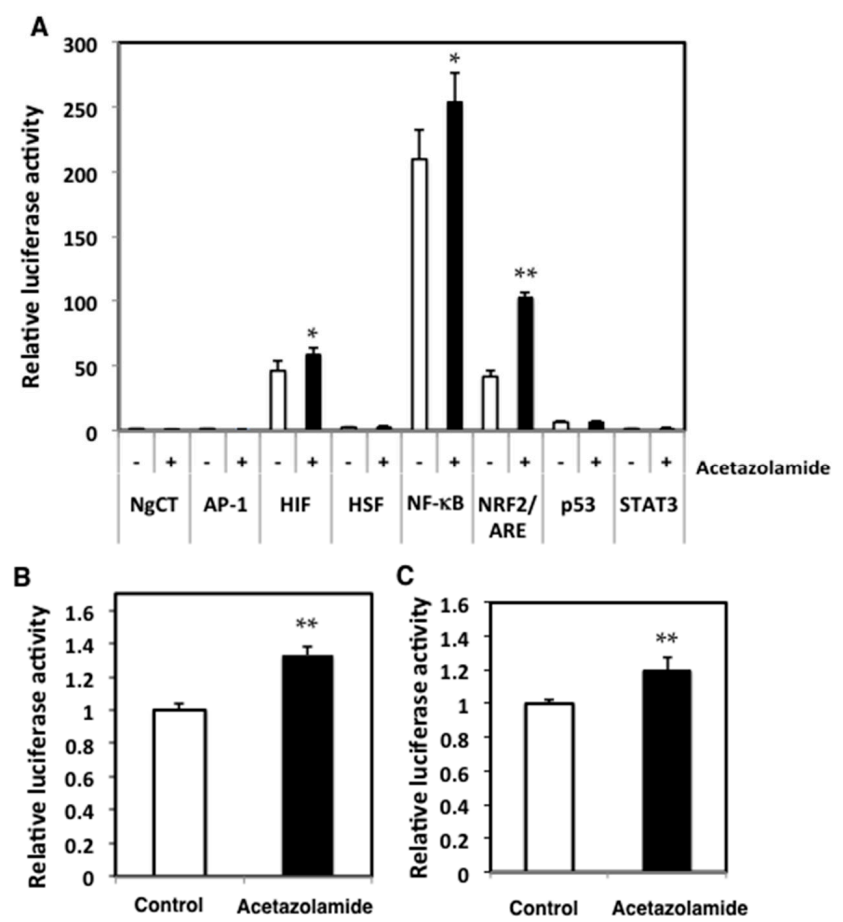

Figure 5. Effects of acetazolamide on the activator protein-1 (AP-1), hypoxia inducible factor (HIF), histamine sensitizing factor (HSF), nuclear factor-kB (NF- $\mathrm{KB}), \mathrm{NRF} 2$, p53, and signal transducer and activator of transcription 3 (STAT3) transcriptional activity in Caco-2 cells (A) with acetazolamide treatment. Values were set at 1.0 in negative control ( $\mathrm{NgCT})$. The change in NRF2 transcriptional activity in human colon cancer cell lines, SW48 cells (B), and HCT15 cells (C), with and without acetazolamide treatment, is shown. The data are given as the mean $\pm \operatorname{SD}(n=3) .{ }^{*} p<0.05,{ }^{* *} p<0.01$ vs. untreated control. 


\subsection{Effects of the Expression Levels of NRF2-Related Factor mRNA in the Intestinal Polyps}

The expression levels of genes regulated by NRF2 were examined in the intestinal polyps by qRT-PCR. Acetazolamide treatment increased the expression levels of $\gamma$-glutamylcysteine synthetase ( $\gamma$ GCS) (1.3-fold), glutathione peroxidase1 (GPx1) (1.6-fold), heme oxygenase-1 (HO-1) (1.3-fold), and $N A D(P) H: q u i n o n e ~ o x i d o r e d u c t a s e-1$ (NQO-1) (1.2-fold) (Figure 6).
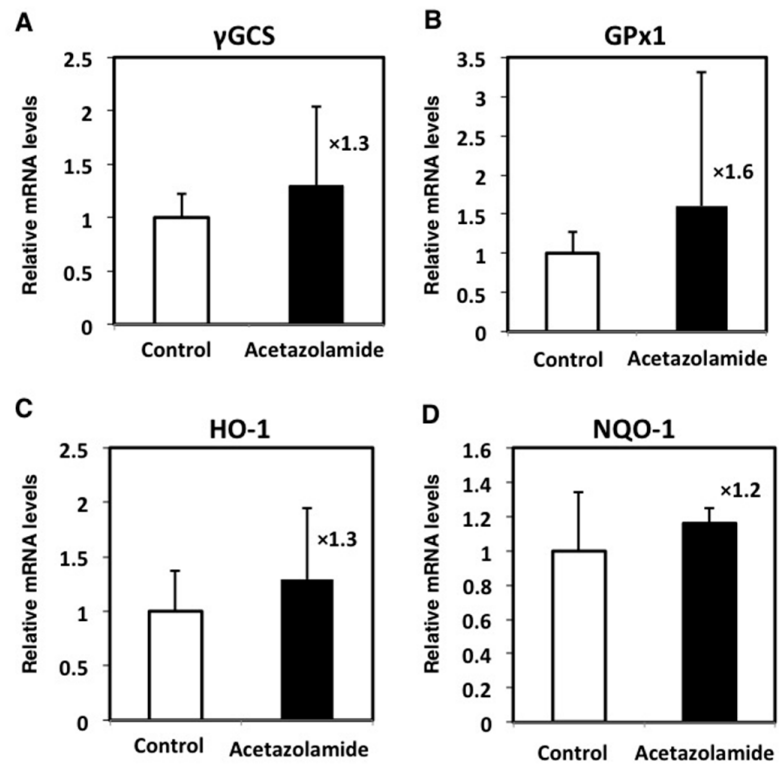

Figure 6. Relative expression levels of NRF2 target genes in intestinal polyps with acetazolamide treatment. The data are given as the mean $\pm \mathrm{SD}(n=5) . \gamma$ GCS, $\gamma$-glutamylcysteine synthetase; GP $x 1$, glutathione peroxidase 1; HO-1, heme oxygenase-1; NQO-1, NAD $(P)$ H:quinone oxidoreductase-1. $\times$ Means multiplication of relative mRNA levels compare to control.

\section{Discussion}

In the present study, we treated Min mice with acetazolamide and observed a reduction in the number of intestinal polyps compared to the control group. In the intestinal polyps of the acetazolamide-treated group, low cell proliferation and a high apoptosis ratio were observed. The proinflammatory cytokine IL-6 mRNA levels were decreased in the intestinal polyps in acetazolamide-treated Min mice. In addition, a reporter gene assay in human colon cancer cells revealed that NRF2 transcriptional activation was strongly induced by acetazolamide, and other transcriptional factors were similarly affected (AP-1, HIF, HSF, NF-kB, NRF2, p53, and STAT3). Moreover, the expression of NRF2-target genes, $\gamma$ GCS, GPx1, HO-1, and NQO1, was elevated in the intestinal polyps of acetazolamide-treated Min mice.

To the best of our knowledge, this is the first report showing that acetazolamide suppressed intestinal polyp formation in mice. As acetazolamide is a pan-CA inhibitor, these data also support the hypothesis that some CA isozymes are involved in intestinal carcinogenesis, and CA could be a good target molecule for cancer chemoprevention.

Our results showed that acetazolamide reduced IL-6 levels in both the local intestinal polyps (i.e., the liver), and the entire mouse (i.e., the serum). IL-6 is a cytokine that is overproduced in chronic inflammation and it could be involved in carcinogenesis. IL-6 activates transcriptional factor STAT3 and upregulates several growth-promoting genes, such as myc and cyclin D1 [13]. In the present study, we observed suppression of $c-M y c$ and cyclin D1 mRNA in the intestinal polyps of Min mice with acetazolamide treatment. Moreover, immunohistochemical staining revealed that acetazolamide treatment reduced the number of PCNA positive cells per field, representing cell growth activity, in the polyps. Therefore, IL-6 reduction is likely involved in the polyp suppressive effects of acetazolamide. 
In another experiment that we previously performed, IL-6 played an important role in the development of intestinal polyps in Min mice [14-16].

NRF2 is a candidate transcriptional factors that may lower the expression levels of IL-6. NRF2 is activated by oxidative stress and binds to the antioxidant response element (ARE) in the promoter region of the genes of cellular antioxidants and phase II enzymes $[17,18]$. Phase II enzymes include $\gamma$ GCS, GPx1, HO-1, and NQO-1. We demonstrated that acetazolamide activates NRF2 transcriptional activity in human colon cancer cells, Caco-2 cells. Indeed, we confirmed that NRF2-targeting genes, $\gamma$ GCS, GPX1, HO-1, and NQO-1, were elevated in the intestinal polyps of Min mice treated with acetazolamide. $\mathrm{CA}$ is responsible for regulating the $\mathrm{pH}$ level, and acetazolamide decreases the intracellular $\mathrm{pH}$ level [8]. Therefore, it is speculated that acetazolamide increased NRF2 activity by inducing oxidative stress through changing the intracellular $\mathrm{pH}$ level, but this speculation requires further experimental evidence. Additionally, NRF2 knockout is known to increase the expression of proinflammatory factors, including IL-6 and MCP-1 [19]. Moreover, NRF2 knockout mice are susceptible to hepatic carcinogenesis [20]. These two articles and our results support the evidence that NRF2 is a critical transcriptional factor that regulates inflammation and carcinogenesis.

In polyps from acetazolamide-treated mice, suppression of $B c l-2$ expression and an increased number of ssDNA positive cells per field were observed. The suppressive effect of acetazolamide on Bcl-2 mRNA expression has not been reported to date. An antiapoptotic protein, Bcl-2, inhibits the release of cytochrome $c$ along with the resultant activation of caspase-9. Caspase-9 cleaves and activates caspase-3, resulting in widespread proteolysis and cell death. Therefore, it is suggested that acetazolamide increases the sensitivity of apoptosis through inhibiting anti-apoptotic Bcl-2.

In summary, this study suggested that acetazolamide inhibits intestinal polyp formation by inducing apoptosis and inhibiting cell growth in Min mice. Inhibition of cell growth could be partially explained by the activation of NRF2 transcriptional activity, resulting in lower IL-6 expression (Figure 7). Although there are still unclear mechanisms to explore, acetazolamide could be a candidate chemopreventive agent.

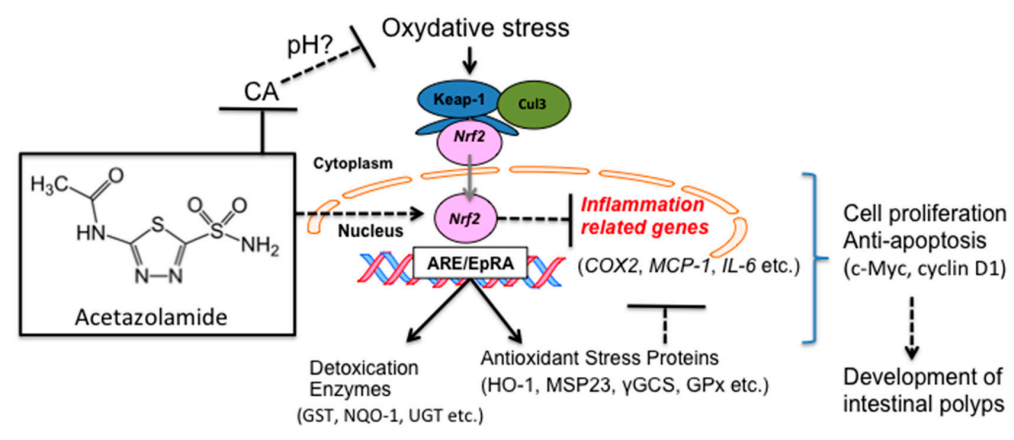

Figure 7. Proposed schema of molecular mechanisms by which acetazolamide suppresses polyp formation in Min mice, partly through the induction of NRF2-transcriptional activity. Solid arrow indicates reliable promoting effect; dotted arrow indicates putative promoting effect; solid T-bar indicates reliable suppressive effect; dotted T-bar indicates putative suppressive effect. Gray arrow indicates nuclear translocation.

\section{Materials and Methods}

\subsection{Chemicals}

Acetazolamide was purchased from Sigma Chemical Co. (St. Louis, MO, USA).

\subsection{Cell Culture}

SW48 and HCT-15 cells were purchased from the American Type Culture Collection (Manassas, VA, USA). Caco-2 cells were purchased from Sumitomo Dainippon Pharma Co., Ltd. (Osaka, Japan). 
SW48 and HCT-15 cells were maintained in Dulbecco's modified eagle medium (DMEM) medium supplemented with 5\% heat-inactivated fetal bovine serum (FBS; HyClone Laboratories Inc., Logan, UT, USA) and antibiotics ( $100 \mu \mathrm{g} / \mathrm{mL}$ streptomycin and $100 \mathrm{U} / \mathrm{mL}$ penicillin) at $37^{\circ} \mathrm{C}, 5 \% \mathrm{CO}_{2}$. Caco-2 cells were maintained in DMEM medium supplemented with $10 \%$ heat-inactivated FBS and antibiotics at $37^{\circ} \mathrm{C}$ and $5 \% \mathrm{CO}_{2}$.

\subsection{Animals}

Male C57BL/6- $A p c^{\mathrm{Min} /+}$ mice (Min mice) were purchased from The Jackson Laboratory (Bar Harbor, ME, USA). Mice $(n=4-5)$ were housed in a plastic cage with sterilized softwood chips as bedding in a barrier-sustained animal room at $24 \pm 2{ }^{\circ} \mathrm{C}$ and $55 \%$ humidity at a 12-h light/dark cycle. Acetazolamide was mixed at concentrations of 200 and $400 \mathrm{ppm}$ in an AIN-76A powdered basal diet (CLEA Japan, Inc., Tokyo, Japan). We calculated doses of 200 and 400 ppm compared to a human dose. The human dose of acetazolamide is $250-1000 \mathrm{mg} /$ day; therefore, we used acetazolamide at an approximately 1.2-2.4-fold higher dose than the human dose.

\subsection{Animal Experiment Protocols}

Ten male Min mice at 5 weeks of age were given 0, 200, and $400 \mathrm{ppm}$ acetazolamide for 8 weeks (Figure 8). All animals in the same cage were in the same treatment group. Food and water were available ad libitum. The animals were observed daily for clinical signs and mortality. The body weight and food consumption were measured weekly. At the time points for sacrifice, mice were anesthetized, and blood samples were collected from the abdominal vein. The intestinal tract was removed and separated into the small intestine, cecum, and colon. The small intestine was divided into the proximal segment ( $4 \mathrm{~cm}$ in length) and the middle and distal segments, halving the remainder of the proximal segment. Polyps in the proximal segments were counted and evaluated under a stereoscopic microscope; the remaining intestinal mucosa (non-polyp portion) was removed by scraping, and the specimens were stored at $-80{ }^{\circ} \mathrm{C}$ for quantitative real-time PCR analysis. Other segments were opened longitudinally and fixed flat between sheets of filter paper in $10 \%$ buffered formalin. The numbers and sizes of polyps and their distributions in the intestine were assessed with a stereoscopic microscope. The experiments were performed according to the "Guidelines for Animal Experiments in the National Cancer Center" and were approved by the Institutional Ethics Review Committee for Animal Experimentation of the National Cancer Center (permission code: T05-022-C11, approval date: 1 April 2014).

A
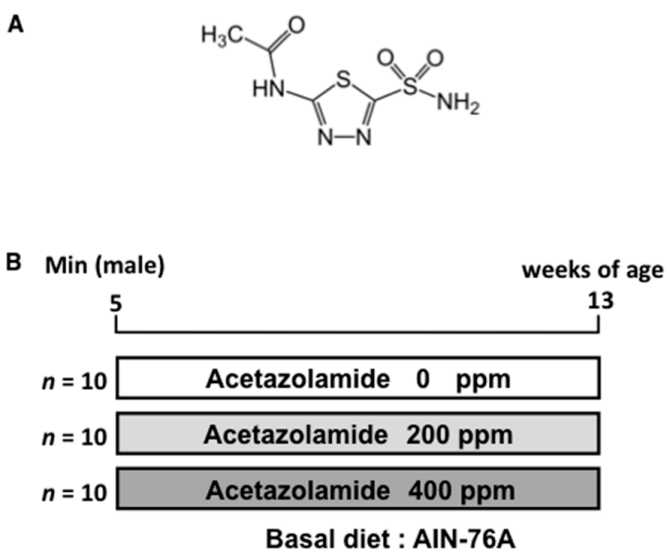

Figure 8. Chemical structure and animal experimental protocol. Structure of acetazolamide (A) and experimental protocol (B). Ten male Min mice at 5 weeks of age were given 0, 200 (0.6 mg/day/mouse), and 400 (1.2 mg/day/mouse) ppm of acetazolamide for 8 weeks. 


\subsection{Immunohistochemical Staining of Intestinal Polyps in Min Mice}

The small intestines were fixed, embedded, and sectioned as Swiss rolls for further immunohistochemical examination using the avidin-biotin complex immunoperoxidase technique after heating in $10 \mathrm{mM}$ citrate buffer ( $\mathrm{pH}$ 6.0). The primary antibody was monoclonal mouse anti-PCNA antibody (Calbiochem, La Jolla, CA, USA) or mouse anti-ssDNA antibody (Chemicon, Temecula, CA, USA) at a $100 \times$ dilution. The secondary antibody, biotinylated horse anti-mouse IgG (Vector Laboratories, Burlingame, CA, USA), was used at a $200 \times$ dilution. Staining was performed using avidin-biotin reagents (Vectastain ABC reagents; Vector Laboratories), 3,3'-diaminobenzidine and hydrogen peroxide, and the sections were counterstained with hematoxylin to facilitate orientation. As a negative control, consecutive sections were immunostained without exposure to the primary antibody. The Swiss roll sections of five mice randomly picked from each group and the total polyps present within one section were evaluated. The ratio of PCNA or ssDNA-positive cells was calculated by the formula $\%=$ the number of PCNA positive cells per polyp/the total number of cells in the polyp $(100 \times$ magnification).

\subsection{Quantitative Real-Time Polymerase Chain Reaction (PCR) Analyses}

Total RNA was isolated from intestinal polyps and non-polyp-containing intestinal mucosa using ISOGEN (Nippon Gene Co., Ltd., Tokyo, Japan); then, it was treated with DNase (Invitrogen, Grand Island, NY, USA) and $1 \mu \mathrm{g}$ in a final volume, or $20 \mu \mathrm{L}$ was used for cDNA synthesis using a High Capacity cDNA Reverse Transcription Kit (Applied Biosystems, Foster City, CA, USA). Real-time PCR was conducted using an MJ Research DNA Engine OPTICON 2 System (MJ Research, Inc., Waltham, MA, USA) and SYBR Green Realtime PCR Master Mix (Toyobo Co., Ltd., Osaka, Japan) according to the manufacturer's instructions. Primer sequences are shown in Table 2. To assess the specificity of each primer set, amplicons generated from the PCR reaction were analyzed for melting curves.

Table 2. Primers for mice.

\begin{tabular}{|c|c|c|}
\hline Gene & & Sequence \\
\hline \multirow[t]{2}{*}{$c-M y c$} & Forward & GCT CGC CCA AAT CCT GTA CCC T \\
\hline & Reverse & TCT CCA CAG ACA CCA CAT CAA TTT C \\
\hline \multirow[t]{2}{*}{ Cyclin D1 } & Forward & TGA CTG CCG AGA AGT TGT GC \\
\hline & Reverse & CTC ATC CGC CTC TGG CAT T \\
\hline \multirow[t]{2}{*}{$B c l-2$} & Forward & ACT TCG CAG AGA TGT CCA GTC A \\
\hline & Reverse & TGG CAA AGC GTC CCC TC \\
\hline \multirow[t]{2}{*}{$I L-6$} & Forward & TGT TCT CTG GGA AAT TCG TGG A \\
\hline & Reverse & AAG TGC ATC ATC GTT GTT CAT ACA \\
\hline \multirow[t]{2}{*}{$M C P-1$} & Forward & CAG CCA GAT GCA GTT AAC GC \\
\hline & Reverse & GCC TAC TCA TTG GGA TCA TCT TG \\
\hline \multirow[t]{2}{*}{ Pai-1 } & Forward & ACG TTG TGG AAC TGC CCT AC \\
\hline & Reverse & GCC AGG GTT GCA CTA AAC AT \\
\hline \multirow[t]{2}{*}{$\gamma G C S$} & Forward & CTA CCA CGC AGT CAA GGA CC \\
\hline & Reverse & CCT CCA TTC AGT AAC AAC TGG \\
\hline \multirow[t]{2}{*}{ GPx1 } & Forward & AAT GTC GCG TCT CTC TGA GG \\
\hline & Reverse & TCC GAA CTG ATT GCA CGG G \\
\hline \multirow[t]{2}{*}{$\mathrm{HO}-1$} & Forward & GAT AGA GCG CAA CAA GCA GAA \\
\hline & Reverse & CAG TGA GGC CCA TAC CAG AAG \\
\hline \multirow[t]{2}{*}{ NQO-1 } & Forward & AGG ATG GGA GGT ACT CGA ATC \\
\hline & Reverse & TGC TAG AGA TGA CTC GGA AGG \\
\hline
\end{tabular}




\subsection{Luciferase Assays for AP-1, HIF, HSF, NF- $k B$, NRF2, $p 53$, and STAT3 Transcriptional Activity}

To measure the AP-1, HIF, HSF, NF- $\mathrm{kB}$, NRF2, p53, and STAT3 transcriptional activity, Caco-2 colon cancer cells were seeded in 96 -well plates $\left(1.0 \times 10^{5}\right.$ cells $/$ well $)$. After a $24 \mathrm{~h}$ incubation period, the cells were transiently transfected with $100 \mathrm{ng} /$ well of pAP1-Luc, pNF-kB-Luc, pNRF2/ARE-Luc, pp53-Luc, pSTAT3-Luc, or pTA-Luc (Signosis Inc., Santa Clara, CA, USA) reporter plasmid and 10 ng/well pGL4.73 [hRluc/SV40] control plasmid (Promega, Madison, WI, USA) using Lipofectamine 2000 Transfection Reagent (Life Technologies, Inc., Gaithersburg, MD, USA). Transfected cells were cultured for an additional $8 \mathrm{~h}$ and treated with $500 \mu \mathrm{M}$ acetazolamide for $24 \mathrm{~h}$. Then, the firefly and Renilla luciferase activities were determined using the Bright GLO and Renilla GLO Systems (Promega), respectively. The ratio of luciferase activity with each treatment was calculated from the data of triplicate wells with values normalized by the Renilla luciferase activity. In HCT-15 or SW48 cells, the NRF2 activity was measured using the same procedure. The data are expressed as the means \pm SD.

\subsection{Enzyme-Linked Immunosorbent Assay (ELISA) for Measuring Murine Serum IL-6 Levels in Mice}

Murine serum IL-6 levels were measured using a Mouse IL-6 Quantikine ELISA Kit (R\&D Systems, Minneapolis, MN, USA) according to the manufacturer's protocol.

\subsection{Statistical Analyses}

All results are expressed as the means \pm SD values, and statistical analyses were performed using Student's $t$-tests. The exceptions are the examination of the body weight, diet intake, organ weight, and intestinal polyp formation/size distribution, which were analyzed by Bonferroni's test. Differences were considered statistically significant at ${ }^{*} p<0.05$ and ${ }^{* *} p<0.01$.

Acknowledgments: We thank Naoaki Uchiya and the National Cancer Center Research Core Facility for contributing to the analyses in this study. The Core Facility was supported by the National Cancer Center Research and Development Fund (23-A-7). This work was supported by Grants-in-Aid for Cancer Research, the Third-Term Comprehensive 10-Year Strategy for Cancer Control from the Ministry of Health, Labour, and Welfare of Japan, and Practical Research for Innovative Cancer Control from the Japan Agency for Medical Research and Development (AMED, 16ck0106098h0003). Shingo Miyamoto was an awardee of the Research Resident Fellowship from AMED during the course of the present research.

Author Contributions: Nobuharu Noma, Sei-ichi Tanuma and Michihiro Mutoh conceived and designed the experiments; Nobuharu Noma, Masami Komiya and Ruri Nakanishi performed the experiments; Gen Fujii, Shingo Miyamoto and Misato Shimura analyzed the data; Nobuharu Noma and Michihiro Mutoh wrote the paper. All authors read and approved the final manuscript.

Conflicts of Interest: The authors declare no conflict of interest.

$\begin{array}{ll}\text { Abbreviations } \\ \text { AP-1 } & \text { Activator protein-1 } \\ \text { ARE } & \text { Antioxidant response element } \\ \text { Bcl-2 } & \text { B-cell lymphoma 2 } \\ \text { CA } & \text { Carbonic anhydrase } \\ \text { CRC } & \text { Colorectal cancer } \\ \text { FBS } & \text { Fetal bovine serum } \\ \gamma \text { GCS } & \gamma \text {-Glutamylcysteine synthetase } \\ \text { GAPDH } & \text { Glyceraldehyde-3-phosphate dehydrogenase } \\ \text { GPx1 } & \text { Glutathione peroxidase 1 } \\ \text { HIF } & \text { Hypoxia inducible factor } \\ \text { HNPCC } & \text { Hereditary nonpolyposis colorectal cancer } \\ \text { HO-1 } & \text { Heme oxygenase-1 }\end{array}$




$\begin{array}{ll}\text { HSF } & \text { Histamine sensitizing factor } \\ \text { IL-6 } & \text { Interleukine-6 } \\ \text { MCP-1 } & \text { Monocyte chemoattractant protein-1 } \\ \text { NF- }- \text { B } & \text { Nuclear factor- } \kappa \text { B } \\ \text { NQO-1 } & \text { NAD(P)H:quinone oxidoreductase-1 } \\ \text { NRF2 } & \text { NF-E2-related factor 2 } \\ \text { Pai-1 } & \text { Plasminogen activator inhibitor-1 } \\ \text { PCNA } & \text { Proliferating cell nuclear antigen } \\ \text { PCR } & \text { Polymerase chain reaction } \\ \text { SSDNA } & \text { Single-stranded DNA } \\ \text { STAT3 } & \text { Signal transducer and activator of transcription 3 }\end{array}$

\section{References}

1. Torre, L.A.; Bray, F.; Siegel, R.L.; Ferlay, J.; Lortet-Tieulent, J.; Jemal, A. Global cancer statistics, 2012. CA Cancer J. Clin. 2015, 65, 87-108. [CrossRef] [PubMed]

2. Sporn, M.B.; Suh, N. Chemoprevention: An essential approach to controlling cancer. Nat. Rev. Cancer 2002, 2, 537-543. [CrossRef] [PubMed]

3. Supuran, C.T. Carbonic anhydrases-An overview. Curr. Pharm. Des. 2008, 14, 603-614. [CrossRef] [PubMed]

4. Supuran, C.T. Carbonic anhydrases: Novel therapeutic applications for inhibitors and activators. Nat. Rev. Drug Discov. 2008, 7, 168-181. [CrossRef] [PubMed]

5. Jarvela, S.; Parkkila, S.; Bragge, H.; Kahkonen, M.; Parkkila, A.K.; Soini, Y.; Pastorekova, S.; Pastorek, J.; Haapasalo, H. Carbonic anhydrase IX in oligodendroglial brain tumors. BMC Cancer 2008, 8, 1. [CrossRef] [PubMed]

6. Chiche, J.; Ilc, K.; Laferriere, J.; Trottier, E.; Dayan, F.; Mazure, N.M.; Brahimi-Horn, M.C.; Pouyssegur, J. Hypoxia-inducible carbonic anhydrase IX and XII promote tumor cell growth by counteracting acidosis through the regulation of the intracellular $\mathrm{pH}$. Cancer Res. 2009, 69, 358-368. [CrossRef] [PubMed]

7. Niemela, A.M.; Hynninen, P.; Mecklin, J.P.; Kuopio, T.; Kokko, A.; Aaltonen, L.; Parkkila, A.K.; Pastorekova, S.; Pastorek, J.; Waheed, A.; et al. Carbonic anhydrase IX is highly expressed in hereditary nonpolyposis colorectal cancer. Cancer Epidemiol. Biomark. Prev. 2007, 16, 1760-1766. [CrossRef] [PubMed]

8. Cianchi, F.; Vinci, M.C.; Supuran, C.T.; Peruzzi, B.; de Giuli, P.; Fasolis, G.; Perigli, G.; Pastorekova, S.; Papucci, L.; Pini, A.; et al. Selective inhibition of carbonic anhydrase IX decreases cell proliferation and induces ceramide-mediated apoptosis in human cancer cells. J. Pharmacol. Exp. Ther. 2010, 334, 710-719. [CrossRef] [PubMed]

9. Akocak, S.; Alam, M.R.; Shabana, A.M.; Sanku, R.K.; Vullo, D.; Thompson, H.; Swenson, E.R.; Supuran, C.T.; Ilies, M.A. PEGylated bis-sulfonamide carbonic anhydrase inhibitors can efficiently control the growth of several carbonic anhydrase IX-expressing carcinomas. J. Med. Chem. 2016, 59, 5077-5088. [CrossRef] [PubMed]

10. Xiang, Y.; Ma, B.; Li, T.; Yu, H.M.; Li, X.J. Acetazolamide suppresses tumor metastasis and related protein expression in mice bearing Lewis lung carcinoma. Acta Pharmacol. Sin. 2002, 23, 745-751. [PubMed]

11. Moser, A.R.; Pitot, H.C.; Dove, W.F. A dominant mutation that predisposes to multiple intestinal neoplasia in the mouse. Science 1990, 247, 322-324. [CrossRef] [PubMed]

12. Su, L.K.; Kinzler, K.W.; Vogelstein, B.; Preisinger, A.C.; Moser, A.R.; Luongo, C.; Gould, K.A.; Dove, W.F. Multiple intestinal neoplasia caused by a mutation in the murine homolog of the APC gene. Science 1992, 256, 668-670. [CrossRef] [PubMed]

13. Yoshimura, A. Signal transduction of inflammatory cytokines and tumor development. Cancer Sci. 2006, 97, 439-447. [CrossRef] [PubMed]

14. Teraoka, N.; Mutoh, M.; Takasu, S.; Ueno, T.; Yamamoto, M.; Sugimura, T.; Wakabayashi, K. Inhibition of intestinal polyp formation by pitavastatin, a HMG-CoA reductase inhibitor. Cancer Prev. Res. 2011, 4, 445-453. [CrossRef] [PubMed]

15. Onuma, W.; Tomono, S.; Miyamoto, S.; Fujii, G.; Hamoya, T.; Fujimoto, K.; Miyoshi, N.; Fukai, F.; Wakabayashi, K.; Mutoh, M. Irsogladine maleate, a gastric mucosal protectant, suppresses intestinal polyp development in Apc-mutant mice. Oncotarget 2016, 7, 8640-8652. [PubMed] 
16. Hamoya, T.; Miyamoto, S.; Tomono, S.; Fujii, G.; Nakanishi, R.; Komiya, M.; Tamura, S.; Toshima, J.; Wakabayashi, K.; Mutoh, M. Chemopreventive effects of a low-side-effect antibiotic drug, erythromycin, on mouse intestinal tumors. JCBN 2017, in press. [CrossRef]

17. Kwak, M.K.; Wakabayashi, N.; Kensler, T.W. Chemoprevention through the Keap1-Nrf2 signaling pathway by phase 2 enzyme inducers. Mutat. Res. 2004, 555, 133-148. [CrossRef] [PubMed]

18. Lee, J.M.; Johnson, J.A. An important role of Nrf2-ARE pathway in the cellular defense mechanism. J. Biochem. Mol. Biol. 2004, 37, 139-143. [CrossRef] [PubMed]

19. Khor, T.O.; Huang, M.T.; Kwon, K.H.; Chan, J.Y.; Reddy, B.S.; Kong, A.N. Nrf2-deficient mice have an increased susceptibility to dextran sulfate sodium-induced colitis. Cancer Res. 2006, 66, 11580-11584. [CrossRef] [PubMed]

20. Aleksunes, L.M.; Manautou, J.E. Emerging role of Nrf2 in protecting against hepatic and gastrointestinal disease. Toxicol. Pathol. 2007, 35, 459-473. [CrossRef] [PubMed]

(C) 2017 by the authors. Licensee MDPI, Basel, Switzerland. This article is an open access article distributed under the terms and conditions of the Creative Commons Attribution (CC BY) license (http:/ / creativecommons.org/licenses/by/4.0/). 